\title{
Plastic Deformation Governed by the Stress Induced Martensitic Transformation in Polycrystals*
}

\author{
By Noboru Ono** and Atsushi Sato***
}

\begin{abstract}
In order to make a quantitative description of the plastic deformation of polycrystalline alloys governed by the stress induced martensitic transformation (SIM), the condition of yield by SIM is formulated for multiaxial stress states. The relation between the plastic strain of a crystallite and the volume fractions of individual martensite variants formed in the crystallite is also given. Owing to its simplicity, the Taylor model is suitable for the present purpose to visualize the essential features of polycrystalline SIM. However, the volume change accompanying the martensitic transformation violates the basic hypothesis of the Taylor model; the homogeneity of strain. This problem is coped with by dealing only with the deviatric stress, deviatric strain and the plastic work done by them in terms of the Taylor model, and then by evaluating the effect of the hydrostatic stress and volume change. This scheme may be useful in predicting the deformation behavior of polycrystalline shape memory alloys, including the effect of their textures.
\end{abstract}

(Received November 6, 1987)

Keywords: stress induced martensitic transformation, polycrystal plasticity, the Taylor model, shape memory alloys

\section{Introduction}

The pseudoelasticity and shape memory effect are known to be caused by the reversetransformation of martensite which has been stress-induced or rearranged by applied stress, and basic mechanismes involved are fairly well understood through a considerable amount of work as reviewed, for instance, in refs. (1) to (4). Especially for the stress-induced martensitic transformation (SIM) observed above $A_{\mathrm{f}}$, it has generally been accepted ${ }^{(1)-(4)}$ that plastic deformation occurs through the nucleation and growth of habit plane variants which have specific orientation relationships to the matrix, and that its critical stress obeys the Schmid law extended to include the effect of the normal stress and changes proportionally to temperature following the Clausius-Clapeyron equation.

* This paper was originally published in Japanese in $\mathbf{J}$. Japan Inst. Metals, 50 (1986), 777.

** Faculty of Engineering, Tohoku University, Sendai 980, Japan.

*** Graduate Student, Tohoku University, Sendai 980. Present address: Sony Magnetic Products Inc., Tagajoh 985, Japan.
In industrial applications, the shape memory alloys exhibiting these phenomena are mostly polycrystalline. The transformation and deformation of polycrystalline shape memory alloys, therefore, have drawn considerable attention; for instance, the grain size dependence of deformation and fracture behavior has been investigated $^{(5)-(8)}$.

A fundamental difference between the deformation of polycrystals from that of single crystals is that the polycrystal grains are subjected to mutual constraint in strain. The significance of this constraint effect on the properties of shape memory alloys has been discussed by Christian ${ }^{(4)}$, but so far little attempt has been made to evaluate this quantitatively.

Following the classical Taylor model ${ }^{(9)}$ and its generalization by Bishop and $\mathrm{Hill}^{(10)}$, various models have been proposed to evaluate the effect of the intergranular constraint in the plastic deformation of polycrystals, e.g., the self-consistent model (KBW model) by Kroener ${ }^{(11)}$ and Budianski and $\mathrm{Wu}^{(12)}$, and Hill's model incorporating the plastic stress relaxation $^{(13)}$. Among these, the Taylor model is a rigid-plastic model and assumes the homogeneity of strain all over a polycrystalline body, so that it is considered to give the upper 
bound of the effect of the intergranular constraint. Based on the comparative studies on various experimental results, however, Kocks has concluded that the Taylor model is sufficiently accurate in predicting the deformation behavior of polycrystals from that of single crystals, although the hypothesis of uniform strain may better be replaced with more realistic ones ${ }^{(14)(15)}$. Further, the observation of deformation structures and the comparison of the observed Bauschinger effect and that predicted on the basis of the KBW model have indicated that the Taylor model well describes the deformation behavior of polycrystals in the strain region above 0.3 to $0.5 \%$, where the development of internal stresses due to the non-uniform strain has come to saturation ${ }^{(16)(17)}$.

As described above, the geometric and thermodynamical features of the stress-induced martensitic transformation are well established in principle, and the Taylor model has been accepted as a good approximation in describing the polycrystal plasticity. Hence, it is considered worthwhile to make an analysis of polycrystalline SIM by use of the Taylor model. The reasons for the use of the Taylor model among the various models mentioned above are: (1) it does not require consideration for the elastic constants, which, in the alloys exhibiting SIM generally, show high anisotropy and change upon transformation ${ }^{(18)}$; (2) the state of the Taylor constraint is considered to be reallized in the strain range from 0.5 to a few per cent, where shape memory alloys are used in most applications; and (3) the simplicity of the Taylor model facilitates the accomodation of the complicated features of SIM into the aimed analysis.

If this analysis is successfully made, the mechanical behavior governed by SIM in single- and polycrystals are related to each other, and thence the results of the basic research on the martensitic transformation are related to the properties of the shape memory alloys in practical use. Also, Bishop and Hill ${ }^{(10)}$ and Kocks ${ }^{(19)}$ have shown in the case of the deformation by slip, the polycrystalline model is capable of constituting the polycrystalline yield surface and its change with the texture.
For SIM also, the same may be possible and this will enable one, for example, to predict the relationship between the results of the tensile test and the SIM in shear deformation of practical interest in coil spring taking into account the effect of the texture.

As the first step toward the above described goal, in the present report, basic formulations will be put forward which describe the uniaxial deformation of polycrystals governed by SIM on the basis of the Taylor model. First, the condition of the stress-induced nucleation and growth of martensite given for single crystals subjected to uniaxial stress ${ }^{(20)}$ are generalized for the case of multiaxial stresses. Next, a scheme is proposed to cope with the inhomogeneous volume change accompanying the polycrystalline SIM, which violates the basic hypothesis of the Taylor model; the homogeneity of strain. With this scheme, the deformation behavior of polycrystalline shape memory alloys by SIM can be predicted from that of single crystals. The accuracy of the prediction can be checked by performing numerical calculations on particular shape memory alloys, and then comparing the results to the corresponding experimental observations. These attempts will be described in the forthcoming papers.

\section{The Yield Surface of Single Crystals}

Here considered is the case that a martensite variant is stress-induced in a single crystal. Some alloys exhibit a large yield drop, while others do not. In the former case, criteria for the nucleation must be considered ${ }^{(21)}$. In the SIM in polycrystals, however, the yield drop is usually not observed. This may be due to the ease of nucleation at the inhomogeneities such as grain boundaries and also due to the stress concentrations originating from elastic anisotropy. Therefore, only the growth process of once nucleated martensite variants is considered hereafter.

When a martensite variant grows under an applied stress, the work done by the stress is expressed as

$$
W=m^{\mathrm{s}} \tau+m^{\mathrm{n}} \sigma_{\mathrm{n}}
$$


Here, $m^{s}$ and $m^{\mathrm{n}}$ are the components of shape strain parallel and normal to the habit plane, respectively, $\tau$ and $\sigma_{\mathrm{n}}$ being the corresponding stress components. Thermodynamical driving force $\Delta G_{\text {chem }}$ also contributes to this process. This is given by the difference between the free energy of the parent phase $G^{\mathrm{P}}$ and that of the martensite $G^{\mathrm{M}}$ in the absence of external stress,

$$
\Delta G_{\text {chem }}(T, P)=G^{\mathrm{P}}-G^{\mathrm{M}}=\Delta U-T \Delta S+P \Omega .
$$

Here, $\Delta U, \Delta S$ and $\Omega$ are the differences between the parent and martensite phases in the internal energy, entropy, and volume, respectively. $T$ and $P$ are temperature and pressure. The net driving force $\Delta G$ is the sum of $W$ and $\Delta G_{\text {chem }}$, and the martensite grows if $\Delta G$ reaches the energy that is irreversibly dissipated upon the growth of martensite $\Delta G_{f}^{(4)(20)}$. That is, the criteria for the martensite growth is given as

$$
\Delta G=\Delta G_{\text {chem }}+W=\Delta G_{\mathrm{f}} .
$$

The yield condition for single crystals is, therefore, given as

$$
W=m^{\mathrm{s}} \tau+m^{\mathrm{n}} \sigma_{\mathrm{n}}=\Delta G_{\mathrm{f}}-\Delta G_{\text {chem }}=W^{*}(T) .
$$

In polycrystalline alloys, grains are subject to mutual constraint in strain, so that, the deformation by SIM requires the simultaneous nucleation and growth of several different variants so as to fulfill the compatibility condition among grains. In order to deal with this situation, it is useful to generalize the above described expression of $W$, as follows.

Let us denote, in an appropriate Cartesian coordinate, the habit plane normal, displacement vector and its magnitude for s'th variants as $h_{\mathrm{i}}^{\mathrm{s}}, d_{\mathrm{i}}^{\mathrm{s}}$ and $\eta$, respectively. The strain of the variant $e_{\mathrm{ij}}^{\mathrm{s}}$ is given, assuming the infinitesimal strain, as

$$
e_{\mathrm{ij}}^{\mathrm{s}}=\frac{1}{2} \eta\left(m_{\mathrm{ij}}^{\mathrm{s}}+m_{\mathrm{ji}}^{\mathrm{s}}\right)
$$

where

$$
m_{\mathrm{ij}}^{\mathrm{s}}=d_{\mathrm{i}}^{\mathrm{s}} h_{\mathrm{j}}^{\mathrm{s}} .
$$

The vector notation is adopted for the 6 independent components of this strain ${ }^{(14)}$ :

$$
\left\{e_{\mathrm{i}}^{\mathrm{s}}\right\}=\left\{e_{11}^{\mathrm{s}}, e_{22}^{\mathrm{s}}, e_{33}^{\mathrm{s}}, 2 e_{23}^{\mathrm{s}}, 2 e_{31}^{\mathrm{s}}, 2 e_{12}^{\mathrm{s}}\right\}
$$

and

$$
\begin{aligned}
\left\{m_{\mathrm{i}}^{\mathrm{s}}\right\}= & \left\{m_{11}^{\mathrm{s}}, m_{22}^{\mathrm{s}}, m_{33}^{\mathrm{s}}, m_{23}^{\mathrm{s}}+m_{32}^{\mathrm{s}},\right. \\
& \left.m_{31}^{\mathrm{s}}+m_{13}^{\mathrm{s}}, m_{12}^{\mathrm{s}}+m_{21}^{\mathrm{s}}\right\} .
\end{aligned}
$$

Similar notation for the stress tensor $\sigma_{\mathrm{ij}}$ is

$$
\left\{\sigma_{\mathrm{i}}\right\}=\left\{\sigma_{11}, \sigma_{22}, \sigma_{33}, \sigma_{23}, \sigma_{31}, \sigma_{12}\right\} .
$$

Thus, the work done by the stress upon the growth of variant $s$ of unit volume, $W$, is written as

$$
W=\sigma_{\mathrm{i}} e_{\mathrm{i}}^{\mathrm{s}} .
$$

The summation convention is applied for suband superscripts, unless otherwise specified. The eq.(1) is the special case of eq. (10), where two coordinate axes are chosen in coincidence with the component of displacement vector parallel to the habit plane and the habit plane normal, respectively. Here, the hydrostatic component of the stress

$$
\sigma_{\mathrm{m}}=\left(\sigma_{1}+\sigma_{2}+\sigma_{3}\right) / 3
$$

is the reverse of pressure, i.e., $\sigma_{\mathrm{m}}=-P$. Further the volume change associated with the transformation $\Omega$ is given, by assuming infinitesimal strain, as

$$
\begin{aligned}
\Omega & =e_{1}^{\mathrm{s}}+e_{2}^{\mathrm{s}}+e_{3}^{\mathrm{s}} \\
& =\eta\left(m_{1}^{\mathrm{s}}+m_{2}^{\mathrm{s}}+m_{3}^{\mathrm{s}}\right) .
\end{aligned}
$$

By using these, eq. (10) may be rewritten in terms of $\sigma_{\mathrm{m}}$ and the deviatric stress and strain, $\sigma_{\mathrm{i}}^{\prime}$ and $e_{\mathrm{i}}^{\prime \text { s }}$, and also $\Omega$, as

$$
W=\sigma_{\mathrm{i}}^{\prime} e_{\mathrm{i}}^{\mathrm{s}}+\sigma_{\mathrm{m}} \Omega .
$$

As seen in this expression, eq. (13), and equivalently eq. (10), contains the term $P \Omega$ in eq. (2). Equation (4) is, therefore, written more appropriately as

$$
W=\sigma_{\mathrm{i}} e_{\mathrm{i}}^{\mathrm{s}}=\Delta G_{\mathrm{f}}-\Delta F_{\text {chem }}=W^{*}(T),
$$

where

$$
\Delta F_{\text {chem }}(T)=\Delta U-T \Delta S .
$$

This gives the condition for the growth of variant $\mathbf{s}$ under a general stress state $\left\{\sigma_{\mathrm{i}}\right\}$.

From these, the yield condition of single crystals by SIM is summarized as

$$
v^{\mathrm{s}} \geqq 0, \quad \text { if } \quad \sigma_{\mathrm{i}} m_{\mathrm{i}}^{\mathrm{s}} \eta=W^{*}
$$

and 


$$
v^{\mathrm{s}}=0, \quad \text { if } \quad \sigma_{\mathrm{i}} m_{\mathrm{i}}^{\mathrm{s}} \eta<W^{*} .
$$

Here, $v^{s}$ is the volume fraction of variant $\mathrm{s}$. In 6 dimentional stress space, eq. (16) constitutes a hyper-polyhedron called the yield surface delineated by the planes of the number of possible variants.

Strain is considered next. As a variant occupies the whole volume of a crystal, the strain of the crystal externally observed, $\varepsilon_{i}$, is apparently given as

$$
\varepsilon_{\mathrm{i}}=e_{\mathrm{i}}^{\mathrm{s}}=\eta m_{\mathrm{i}}^{\mathrm{s}} .
$$

The strain produced by the formation of different variants is

$$
\varepsilon_{\mathrm{i}}=\eta v^{\mathrm{s}} m_{\mathrm{i}}^{\mathrm{s}}
$$

where

$$
v=\Sigma v^{s} \leqq 1 .
$$

Here, $v$ is the volume fraction of martensite. The martensitic transformation is generally accompanied by a volume change, whose magnitude is given by $\Omega$ in eq. (12), independent of the variants. The volume change of a crystal, $\Delta V$, containing martensite of a volume fraction $v$ is given as

$$
\Delta V=\Omega v .
$$

\section{Polycrystal Model}

When a polycrystalline aggregate undergoes an overall strain $\bar{\varepsilon}_{\mathrm{i}}$, the Taylor model assumes that the strain in individual grains $\varepsilon_{\mathrm{i}}$ is homogeneous, being equal to $\bar{\varepsilon}_{\mathrm{i}}$, i.e.,

$$
\varepsilon_{\mathrm{i}}=\eta v^{\mathrm{s}} m_{\mathrm{i}}^{\mathrm{s}}=\bar{\varepsilon}_{\mathrm{i}},
$$

by using eq. (18).

In the case of the deformation by slip which does not accompany the volume change, the number of independent components of $\bar{\varepsilon}_{\mathrm{i}}$ is 5 , so that the operation of at least 5 independent slip systems is required for the compatibility condition of strain similar to eq. (21) to be satisfied. This, in turn, requires that the stress state called the corner or the vertex $\sigma_{\mathrm{i}}^{\mathrm{c}}$ is attained, where more than 5 planes cross each other on the yield surface corresponding to eq. (16). If a strain $\bar{\varepsilon}_{\mathrm{i}}$ is prescribed, a vertex satisfying the yield condition, eq. (16), is specified uniquely for each grain orientation, and the polycrystalline yield stress is given by its average for a grain orientation distribution. In the case of polycrystalline SIM, however, the associating volume change produces some problems to be coped with.

Again, considering the uniaxial deformation by slip, the ratio of the axial component of the vertex stress $\sigma_{1}^{\mathrm{c}}$ to the critical resolved shear stress $\tau_{\mathrm{c}}$ is called the Taylor factor, $M$, whose magnitude varies depending on grain orientation $^{(9)(14)}$. The same factor correlates the axial strain $\varepsilon_{1}$ to the sum of the shears of all active slip systems, $\Gamma$, as $\varepsilon_{1}=\Gamma / M$. In the case of SIM, as described below, a similar relationship holds; the volume fraction of martensite $v$ corresponds to $\Gamma$ in the slip deformation, and similarly $v$ for a same axial strain varies from grain to grain. According to eq. (20), therefore, the amount of volume change differs from grain to grain, depending on their orientation. Thus, the strain compatibility condition, eq. (21), cannot be fulfilled in SIM that accompanies volume change, so that the Taylor model cannot be adopted strictly. In reality, the existence of elastic accommodation of strain mismatch permits polycrystals to undergo ductile deformation, where the internal hydrostatic stresses due to the inhomogeneity in volume change balance against the axial internal stresses due to the inhomogeneity in axial strain. The internal stresses as such cannot be incorporated in the Taylor model and the evaluation of their magnitude and their effects requires an elasto-plastic model such as the KBW model. In order to avoid the complexity associating with this, a modification of the Taylor model is devised as described in the following.

First, according to eq. (13), the plastic work done by the stress in the left hand side of eq. (16) is divided into the work done by deviatric stress and that by hydrostatic stress. Taking out the former, one gets an expression of the yield condition which does not explicitly contain presently unknown hydrostatic stress:

where

$$
\sigma_{\mathrm{i}}^{\prime} m_{\mathrm{i}}^{\mathrm{s}} \leqq W^{\prime}\left(T, \sigma_{\mathrm{m}}\right) / \eta
$$

$$
W^{\prime}\left(T, \sigma_{\mathrm{m}}\right)=W^{*}(T)-\sigma_{\mathrm{m}} \Omega .
$$


Multiply both sides of this equation by $v^{\mathrm{s}}$ and sum up for s. Note that the equality holds only for $\mathrm{s}$ with non-zero $v^{\mathrm{s}}$. With this and eq. (18), one gets

$$
\sigma_{\mathrm{i}}^{\prime} \varepsilon_{\mathrm{i}}=W^{\prime} v .
$$

The left hand side is equivalent to

$$
\sigma_{\mathrm{i}}^{\prime} \varepsilon_{\mathrm{i}}^{\prime}=W^{\prime} v \text {. }
$$

Here, from the assumption in the Taylor model, $\varepsilon_{4}=\varepsilon_{5}=\varepsilon_{6}=0$. Also it holds that

$$
\sigma_{2}^{\prime}+\sigma_{3}^{\prime}=-\sigma_{1}^{\prime},
$$

and

$$
\varepsilon_{2}^{\prime}=\varepsilon_{3}^{\prime}=-\varepsilon_{1}^{\prime} / 2 .
$$

Equation (24) is, therefore, reduced to

$$
\frac{3}{2} \sigma_{1}^{\prime} \varepsilon_{1}^{\prime}=W^{\prime} v \text {. }
$$

From this relationship, which has been derived by eliminating the effects of both volume change and hydrostatic stress, we obtain a "modified" definition of the Taylor factor as follows:

$$
M^{\prime}=\frac{3}{2} \eta \sigma_{1}^{\prime} / W^{\prime}=\eta v / \varepsilon_{1}^{\prime} .
$$

$\sigma_{1}^{\prime}$ in this equation satisfies the yield condition, eq. (22), so that $M^{\prime}$ thus defined is a geometrical factor depending only on grain orientation. In the deformation of real polycrystals, of interest are the relation between $\sigma_{1}$ and $W^{*}$ and that between $\varepsilon_{1}$ and $v$, both including the effects of the ignored hydrostatic component and volume change, so that further consideration is needed.

Suppose a grain is subjected to a hydrostatic stress $\sigma_{\mathrm{m}}$. From eqs. (22b) and (28),

$$
\sigma_{1}^{\prime}=\frac{2}{3} M^{\prime}\left(W^{*}-\sigma_{\mathrm{m}} \Omega\right) / \eta .
$$

By averaging this, $\bar{\sigma}_{1}^{\prime}$ for the polycrystalline aggregates as a whole is given as

$$
\bar{\sigma}_{1}^{\prime}=\frac{2}{3}\left(\overline{M^{\prime}} W^{*}-\Omega \overline{M^{\prime} \sigma_{\mathrm{m}}}\right) / \eta
$$

One may rewrite the second term in the braket by putting $M^{\prime}=\overline{M^{\prime}}+\Delta M^{\prime}$ and $\sigma_{\mathrm{m}}=\bar{\sigma}_{\mathrm{m}}+\Delta \sigma_{\mathrm{m}}$. Equation (30) then becomes

$$
\bar{\sigma}_{1}^{\prime}=\frac{2 M^{\prime}}{3 \eta} W^{*}-\frac{2 \Omega}{3 \eta} \bar{M}^{\prime} \bar{\sigma}_{\mathrm{m}}-\frac{2}{3} \overline{\Delta M^{\prime} \Delta \sigma_{\mathrm{m}}} .
$$

Here, $\Delta \sigma_{\mathrm{m}}$ results from the inhomogeneity in the volume change from grain to grain. In order to evaluate this, it is needed to step out of the scheme of the Taylor model: As is the case with the KBW model, we assume that a grain with a volume change $\Delta V$ is embedded in a uniform matrix with a volume change $\overline{\Delta V}$. If the elastic isotropy is assumed for the sake of simplicity, the hydrostatic internal stress produced in the grain is, from the solution of Eshelby $^{(22)}$, given as

$$
\Delta \sigma_{\mathrm{m}}=-G^{\prime}(\Delta V-\overline{\Delta V}),
$$

where $G^{\prime}$ is given, with the shear modulus $\mu$ and Poisson's ratio $v$ as

$$
G^{\prime}=\frac{4}{9} \mu \frac{1+v}{1-v} .
$$

Further using eqs. (20) and (28),

$$
\Delta \sigma_{\mathrm{m}}=-G^{\prime} \frac{\Omega}{\eta} \varepsilon_{1}^{\prime} \Delta M^{\prime} .
$$

The third term of eq. (31), $h$ is then reduced to

$$
h=\frac{2}{3} G^{\prime}(\Omega / \eta)^{2} \overline{\Delta M^{\prime 2}} \varepsilon_{1}^{\prime},
$$

representing a linear work hardening. The magnitude of the hardening is proportional to $\overline{\Delta M^{\prime 2}}$, and thus unknown before the calculation of $M^{\prime}$ is performed on particular alloys. From this, and also because $h$ does not influence the yield stress, it is ignored for the moment.

Equation (31) then becomes

$$
\overline{\sigma_{1}^{\prime}}=\frac{2 \overline{M^{\prime}}}{3 \eta} W^{*}-\frac{2 \Omega}{3 \eta} \overline{M^{\prime}} \bar{\sigma}_{\mathrm{m}} .
$$

Applying the relations among $\bar{\sigma}_{\mathrm{m}}, \bar{\sigma}_{1}$ and $\bar{\sigma}_{1}^{\prime}$ in uniaxial deformation,

$$
\bar{\sigma}_{\mathrm{m}}=\frac{1}{3} \bar{\sigma}_{1}=\frac{1}{2} \bar{\sigma}_{1}^{\prime},
$$

one gets,

$$
\bar{\sigma}_{1}=\bar{M} W^{*} / \eta,
$$

and 


$$
\bar{M}=\overline{M^{\prime}}\left(1+\frac{\overline{M^{\prime} \Omega}}{3 \eta}\right)^{-1} .
$$

Here, $\bar{M}$ is the average Taylor factor for the polycrystalline SIM, including the effect of hydrostatic stress component. In this way, the uniaxial yield stress of a polycrystalline alloys by SIM, $\bar{\sigma}_{1}$, is related to the critical work for the martensite growth $W^{*}$, with the geometrical characteristics of the martensite, $M^{\prime}, \Omega$ and $\eta$.

The axial strain $\varepsilon_{1}$, on the other hand, is given by adding the contribution of the volume change to $\varepsilon_{1}^{\prime}$ as

$$
\varepsilon_{1}=\varepsilon_{1}^{\prime}+\frac{1}{3} \Delta V=\varepsilon_{1}^{\prime}\left(1+\frac{M^{\prime} \Omega}{3 \eta}\right) .
$$

Considering the so far assumed homogeneity in $\varepsilon_{1}^{\prime}$, one may average this to obtain

$$
\bar{\varepsilon}_{1}=\varepsilon_{1}^{\prime}\left(1+\frac{\overline{M^{\prime}} \Omega}{3 \eta}\right) .
$$

This, along with the average of eq. (28) $\varepsilon_{1}^{\prime} \overline{M^{\prime}}=\eta / \bar{v}$ and the definition of $\bar{M}$ in eq. (37), results in

$$
\bar{\varepsilon}_{1}=\eta \bar{v} / \bar{M} .
$$

Thus, the axial strain $\bar{\varepsilon}_{1}$ and the average volume fraction of martensite $\bar{v}$ is related to each other by $\bar{M}$. From eqs. (36) and (40), one get a relation

$$
\bar{\sigma}_{1} \bar{\varepsilon}_{1}=W^{*} \bar{v},
$$

which shows that the work done by the applied stress is in balance with the work dissipated for the formation of martensite.

\section{Summary}

For the stress induced martensitic transformation, the yield condition, eq. (22), has been constituted from the knowledge of its crystallographic data such as the habit plane normal, displacement vector and its magnitude. This and the constraint condition that $\varepsilon_{\mathrm{i}}^{\prime}$ be homogeneous give the "modified" Taylor factor $M^{\prime}$ in eq. (28), similarly to the case of slip deformation. The thermodynamic data $W^{*}$ may be obtained from single crystal experiments with eq. (4). With all these, polycrystalline yield stress is predicted as shown in eq. (36). Here, the information on the grain orientation distribution may be taken into account upon the averaging of $M^{\prime}$, so as to evaluate the effect of crystallographic texture on the SIM.

In this way, the value of $M^{\prime}$ and its grain orientation dependence have direct influence on the deformation behavior of polycrystalline shape memory alloys by SIM. $M^{\prime}$ has been defined through eq. (28) as a factor that relates $\sigma_{1}^{\prime}$ and $\varepsilon_{1}^{\prime}$ to $W^{\prime}$ and $v$, respectively. The average Taylor factor $\bar{M}$ that relates observed axial stress and strain, $\bar{\sigma}_{1}$ and $\bar{\varepsilon}_{1}$, to the true yield strength $W^{*}$ and the average volume fraction of martensite $\bar{v}$, is not the simple average of $M^{\prime}$. This is because the martensitic transformation is influenced by hydrostatic stress, which comes from the axial applied stress $\bar{\sigma}_{1}$, apart from those produced by the inhomogeneity in volume change. $\bar{\sigma}_{1}$ for a $W^{*}$ is influenced by the grain orientation distribution, the factor relating between $\sigma_{1}$ and $W^{*}$ cannot be defined uniquely from the grain orientation alone.

It is of interest how significant are the internal stresses caused by the inhomogeneity in volume change, as given by eq. (33), and also its effect on the flow stress, as given by eq. (34). Along with the linear work hardening according to eq. (34), $\Delta \sigma_{\mathrm{m}}$ in individual grains may directly enhance the grain boundary fracture.

Equations (28) and (38) results in an expression of strain in individual grains;

$$
\varepsilon_{1}=\frac{\eta}{M^{\prime}}\left(1+\frac{M^{\prime} \Omega}{3 \eta}\right) v .
$$

The martensite volume fraction $v$ cannot exceed the unity, so that this equation gives the maximum strain produced by SIM in a grain of an orientation under the intergranular constraint. Thus, this and its average, eq. (40), are considered to give measures the maximum reversible strain in a shape memory alloy.

As described above, the present scheme may be useful in industrial applications of shape memory alloys, providing a measure to correlate the basic mechanisms of martensitic transformation to the deformation behavior of polycrystalline shape memory alloys, quan- 
titatively. The examination on the accuracy of this scheme requires calculation of $M^{\prime}$ on alloys for which crystallographic data are known and also the comparison of the calculated results to systematic experimental results. Such investigation is now under the way.

\section{Acknowledgments}

The authors wish to express their gratitude to the late Prof. T. Homma for his helpful advice on this work. Discussion with Prof. M. Watanabe and Dr. Y. Shugo is gratefully acknowledged.

\section{REFERENCES}

(1) L. Delaey, R. V. Krishnan, H. Tas and H. Warlimont: J. Mater. Sci., 9 (1974), 1521, 1536, 1545.

(2) J. Perkins (ed.): Shape Memory Effects in Alloys, Plenum Press, New York, (1975).

(3) K. Otsuka and K. Shimizu: Metals Forum, 4 (1981), 142.

(4) J. W. Christian: Metall. Trans., 13A (1982), 509.

(5) K. Enami, N. Takimoto and S. Nenno: J. de Phys., C4-43 (1982), 773.

(6) S. M. White, J. M. Cook and W. M. Stobbs: J. de Phys., C4-43 (1982), 779.

(7) G. N. Sure and L. C. Brown: Metall. Trans., 15A (1984), 1613.

(8) T. Saburi and S. Nenno: Proc. Int. Conf. Solid-Solid
Phase Transformation, H. I. Aaronson et al., (eds.), AIME, (1981), p. 1455.

(9) G. I. Taylor: J. Inst. Metals, 62 (1938), 307.

(10) J. F. W. Bishop and R. Hill: Phil. Mag., 42 (1951), 414, 1298.

(11) E. Kroener: Acta Metall., 9 (1961), 155.

(12) B. Budianski and T. T. Wu: Proc. 4th U. S. Nat'l. Congr. Appl. Mechanics, R. M. Rosenberg(ed.), ASME, New York, (1962), p. 1175.

(13) R. Hill: J. Mech. Phys. Solids, 13 (1965), 89.

(14) U. F. Kocks: Metall. Trans., 1 (1970), 1121.

(15) U. F. Kocks and G. R. Canova: Deformation of Polycrystals: Mechanics and Microstructures, N. Hansen et al. (eds.), Riso Nat. Lab., Denmark, (1981), p. 35 .

(16) N. Ono and S. Karashima: Scr. Metall., 16 (1982), 149.

(17) N. Ono, T. Tsuchikawa, S. Nishimura and S. Karashima: Mater. Sci. Engg., 59 (1983), 223.

(18) M. Yasunaga, Y. Funatsu, S. Kojima, K. Otsuka and T. Suzuki: J. de Phys., C4-43 (1982), 603.

(19) U. F. Kocks: Unified Constitutive Equations for Plastic Deformation and Creep of Engineering Alloys, A. K. Miller(ed.), Elsevier, New York, (1985), chap. 2.

(20) J. R. Patel and M. Cohen: Acta Metall., 1 (1953), 531.

(21) K. Otsuka, C. M. Wayman, K. Nakai, H. Sakamoto and K. Shimizu: Acta Metall., 24 (1976), 207.

(22) J. D. Eshelby: Elastic Inclusions and Inhomogeneities, Progress in Solid Mechanics, I. N. Sneddon and R. Hill, (eds.), North-Holland, Amsterdam, 2 (1961), p. 89. 